\title{
Development of A Literacy-Appropriate Health Education Package to Create Awareness of Periodontal Disease and Diabetes
}

\author{
Shahida Mohd-Said ${ }^{1}$, Nur Liyana Mohanad Zaimi', Muhammad Razwan Ruslan ${ }^{1}$, \\ Muhammad Azuan Bin Mohd Zain ${ }^{1}$, Tuti Ningseh Mohd-Dom²
}

\author{
${ }^{1}$ Department of Periodontology, Faculty of Dentistry, Universiti Kebangsaan Malaysia, Jalan Raja Muda Abdul \\ Aziz, 50300 Kuala Lumpur, Malaysia \\ ${ }^{2}$ Department of Dental Public Health, Faculty of Dentistry, Universiti Kebangsaan Malaysia, Jalan Raja Muda \\ Abdul Aziz, 50300 Kuala Lumpur, Malaysia \\ Correspondence e-mail to: shahidams@gmail.com
}

\begin{abstract}
Low oral health literacy has been associated with poor awareness and understanding of oral health care needs among diabetes patients. Specifically, the association between diabetes and periodontal disease is not known to them. Objective: To develop a literacy-appropriate health education package to create public awareness on periodontal disease and diabetes. In spite of the rising prevalence of both diseases, many people remain unaware of their early signs and relationship with each other. Methods: We divided the study into three phases: needs assessment, development of the package and evaluation of the package. Phase 1 was conducted via a Focus Group Discussion (FGD) involving periodontitis patients with diabetes recruited from the UKM Dental Faculty patient list. For phase 2, we developed three video clips of 5-minutes duration each, based on the themes that emerged from the FGD. Phase 3 was conducted using a set of self-administered questionnaires distributed to the public and patients. Results: Sixty respondents viewed the videos and at least $95 \%$ found them interesting, easily understood, useful and satisfactory. There were however some suggestions for improvement. Conclusion: The health education package developed in this study was literacy-appropriate for the general public and should be made accessible to them.
\end{abstract}

Keywords: diabetes, educational video, health literacy, periodontal disease

\section{INTRODUCTION}

Low oral health literacy has been associated with poor awareness and understanding of oral health care needs among patients with diabetes. ${ }^{1}$ Specifically, the association between diabetes and periodontal disease is not known to them. ${ }^{2,3}$ Oral health literacy can be defined as "the degree to which individuals have the capacity to obtain, process, and understand basic oral health information and services needed to make appropriate health decisions". ${ }^{4}$ It has also been associated with poor oral health status among the general population. ${ }^{5}$

The relationship between periodontal disease and diabetes mellitus has been studied extensively from the earlier decades - providing us with increasing evidence of profound two-way relationship between them. ${ }^{6,7}$ For decades now, dentists recognise periodontal disease as the sixth complication of diabetes mellitus and more prevalent in patients with diabetes mellitus than those without diabetes (81.1 versus 17.0 new cases/1000 person per year respectively). ${ }^{8}$ Furthermore, patients with periodontal disease generally demonstrate a low level of knowledge on various aspects of periodontal disease. ${ }^{9}$ Subsequently patients will only realize that they may have periodontal disease when more severe symptoms arise, such as teeth becoming loose and having to be extracted. ${ }^{10}$ This is worrying as the prevalence of periodontal disease, including periodontitis, among adults in Malaysia is increasing, as also the prevalence of diabetes. ${ }^{11,12}$ 
One of the ways to reduce oral health disparities and improve the quality of dental care is by improving public's oral health literacy. ${ }^{13}$ Some efforts that have been shown to improve diabetes patients' health literacy include the use of multimedia presentation and counseling. ${ }^{14,15}$ However, while such multimedia educational program interventions showed increases in the diabetes knowledge among the patients, they do not fill the gaps of knowledge between patients with low and high literacy. This means that although the video presentation may be successful in conveying its messages to the high literacy patients, they did not benefit those with low literacy levels. Thus, this study aimed to develop a literacy-appropriate health education package to create awareness on periodontal disease and diabetes among the general public of all levels of literacy, with special consideration for those with inadequate literacy.

\section{METHODS}

The Universiti Kebangsaan Malaysia's (UKM) Institutional Review Board for Research and Ethics approved this study (No: UKM-SPPI-BO02 dated $15 / 5 / 2014$ ). It is a prospective study involving evaluation of an intervention. We have chosen to adopt a combination of qualitative methods for data collection via a focus group discussion (FGD) and interviews, and quantitative methods for evaluation of the health education package. One advantage of using the qualitative method is that it helps to ensure that patients with low literacy level could understand the questions as clarifications may be made between the interviewer and interviewee. ${ }^{16}$ We divided our method of study into three phases: phase I - need assessment, phase II -development of oral health education package and phase III - evaluation of an oral health education package.

The needs assessment was done to identify patients' gaps in knowledge regarding periodontitis, diabetes, and the relationship between these two diseases. We conducted an FGD involving six patients who were conveniently selected from the list of patients provided by the Periodontology Department at the Faculty of Dentistry, Universiti Kebangsaan Malaysia. This study site is located in the city centre of Kuala Lumpur which is highly accessible by various modes of land transportation. These patients met our selection criteria. Inclusion criteria for selection of respondents were all active periodontitis patients who have diabetes (controlled and uncontrolled) between 2011 and 2014. Exclusion criteria were patients who were not contactable, refused to participate illiterate and who were non-Malaysians. We contacted respondents via telephone to gauge their interest in participating in the study before inviting them to the FGD session.
The FGD took about three hours with a twenty- minute break in between. A trained facilitator conducted the discussion using a set of guided questions. We recorded the session using two digital audio-recorders and obtained participants' consent before commencing the discussion. We conducted face-to-face interviews with three other patients on separate occasions as they were not able to attend the FGD. The interviews took about one hour per session. This was also to ensure that the saturation stage of data acquisition had been achieved. We consolidated and analysed the themes which emerged from the FGD and interviews before starting phase II of the study.

The development of an oral health education (OHE) package was done according to the findings of the focus group discussion. We developed and produced a three-video health education series based on the themes which emerged from FGD. The videos were based on interviews of dental and medical specialists and researchers, and patients who had both periodontitis and diabetes. We followed guidelines from the The Patient Education Materials Assessment Tool (PEMAT) prepared by the Agency for Healthcare Research and Quality of the U.S. Public Health Service. ${ }^{17}$ This tool provides a systematic method to evaluate and compare the understandability and actionability of patient education materials. It is designed as a guide to help determine whether patients will be able to understand and act on information. We had used the tool developed for audiovisual materials.

An evaluation of the oral health education package was done after the video prototypes was finalised. The package consisted of three videos: (1) What is periodontal disease, (2) Periodontitis and diabetes, and (3) Periodontitis, diabetes and you. We evaluated the oral health education package using self-administered close-ended questionnaires which we distributed to the public and patients using convenient sampling. Respondents who were patients were interviewed at the UKM dental clinics, while the public who were interviewed were those approached at areas nearby the dental school. There was one open-ended question asking for suggestions to improve the videos. The sample size for evaluation was 60 respondents.

\section{RESULTS}

\section{Participants' personal profile}

Participants of the FGD and interviews were $66.67 \%$ males and $33.33 \%$ were females. Their mean age was 53.7 years old and median age was 50 years old. The themes which emerged from the FGD were patients' experiences related to periodontitis and diabetes, their oral health literacy, communication between oral health and diabetes, and access to oral health care (Table 1). 
Table 1. Themes and sub-themes from the analysis of focus group discussion

\begin{tabular}{l}
\hline Experiences related to periodontal health and diabetes \\
Symptoms of periodontal disease \\
Experiences with previous dental visits \\
Symptoms of diabetes \\
\hline Relationship between oral health and diabetes \\
No previous awareness and knowledge of connection \\
between oral health and diabetes \\
\hline Oral health literacy \\
Do not understand language, jargon, medical/ dental terms \\
used in communications \\
Do not make connection or change behaviour due to lack \\
of understanding of needs \\
Do not like to read written materials to receive health \\
information \\
\hline Professional oral health care \\
Referrals by medical doctors \\
Reasons for choosing UKM Dental Clinics - easy access, \\
minimal cost \\
Previous experience at private dental clinics \\
Satisfaction with student clinician
\end{tabular}

\section{Experiences related to periodontal health and diabetes}

None of the FGD participants had ever heard of the word 'periodontitis' prior their visit to the UKM periodontics clinics. Symptoms related to periodontitis experienced by the participants include gum bleeding, gum recession, gum inflammation and looseness of teeth. Earlier they were not aware that these were symptoms of periodontal disease and so did not seek treatment earlier. Even for those who had earlier sought treatment outside the UKM dental clinics, the dentists did not explain that they had periodontal disease.

In relation to diabetes, some of the participants claimed that they started to suspect that they may have diabetes because they experienced symptoms that were portrayed in the Health Ministry messages on television. Having symptoms such as excessive hunger and thirst, weight loss, fatigue and frequent urination prompted them to obtain a medical examination which then confirmed that they have diabetes.

\section{Relationship between oral health and diabetes}

All participants did not know about the connection between oral health and diabetes. This includes the relationship between diabetes and periodontitis. It was not surprising then to learn that they did not know of the bidirectional relationship between these two diseases. Now that they are receiving treatment for both diabetes and periodontal disease, they understand their responsibility for daily management of their diabetes and oral health care.

\section{Oral health literacy}

Most of the participants were aware on the importance of having good oral health. This information was received from various sources such as the internet, dentists, periodontologists, oral health education messages on the television and also through their daily conversation with peers. However, throughout the discussion, the participants voiced their frustration that sometimes they could not understand dental terms that were used by their clinicians when explaining about dental treatment. They are not sure of what they are supposed to do for self-care and what to expect during dental treatment. They realised that the public awareness about gum disease is very low and therefore there is a need to provide materials that are easy to be understood by the public.

When being asked regarding what are the sources of information that they preferred, they suggested use of the internet, multimedia presentations, dentist advice, radio, Whatsapp/Android Application, posters, mass media, monitor screens at clinics' waiting area and also appointing celebrities as oral health ambassadors to convey information on oral health. They all in unison stated that they were not keen to read educational brochures or pamphlets as these were considered too wordy and also difficult to read and understand.

\section{Professional oral health care}

Some of the participants were referred to the UKM dental clinic by the medical doctors who were treating them for diabetes in UKM Medical Center. Easy access to oral health care and minimal cost are the main reasons why the patients preferred to seek treatment at the UKM dental clinics. One of the participants had previously received dental treatment in a private clinic and complained that the service was bad and the cost was very expensive. One of the participants who described himself as a dental phobic said that he only came to have dental treatment when he could not bear the dental pain. He was happy to share that since he started receiving treatment at the UKM dental clinic, his dental needs had been addressed appropriately. All patients were satisfied with dental treatment provided by UKM students.

\section{Evaluation of the health education videos}

Sixty respondents viewed the videos and provided their feedback on them (Table 2). The mean age of the respondents was $29.3 \pm \mathrm{SD} 11.8$ year. There was an almost equal proportion between those who had secondary school and college/university as their highest level of education, and also between those who were working and still studying. About two-thirds were not patients of UKM dental clinics. 
Feedback on the videos is shown in Table 3. For video 1 entitled 'What is periodontal disease?', 90\% respondents agreed that it was interesting, 98\% agreed it was easily understood, $100 \%$ agreed that the information provided was useful and 95\% were satisfied with it. For video 2 entitled 'Periodontitis and Diabetes', 92\% respondents agreed that it was interesting, 90\% agreed that it was easily understood, $100 \%$ agreed that the information provided was useful, and 95\% were satisfied with it. For video 3 entitled 'Periodontitis, Diabetes and You', 97\% respondents agreed that it was interesting. 95\% agreed that it was easily understood, 98\% agreed that the information provided was useful and $95 \%$ were satisfied.

For the open-ended question, respondents made specific comments to improve the videos: to shorten their duration, to improve the audio-visual quality, to explain more the medical/ dental terms used, to make the layout more attractive, and the need for further elaboration of information (Table 4).

\section{DISCUSSION}

This study was done to develop and produce a set of literacy-appropriate health education materials on periodontal disease and diabetes for use by the Malaysian population of various literacy levels. We conducted an FGD involving periodontitis patients with diabetes who were receiving treatment at the UKM Faculty of Dentistry clinics. From the FGD, four themes emerged: patients' experiences related to periodontitis and diabetes, relationship between oral health and diabetes, oral health literacy and professional oral health care. These findings are consistent with what is available in the literature. ${ }^{1-3}$

All FGD participants knew about signs and symptoms of diabetes. They experienced it and could relate the sign and symptoms to their condition based on information they received from various sources even before seeking for treatment. There were cues to action, such as watching health education videos on diabetes. As for periodontitis, they had little knowledge about it. None of them ever heard of word periodontitis, more so about the signs and symptoms, until after they were given oral health education by their student clinicians as the Dental Faculty. They experienced the disease without realising that these were symptoms of periodontal disease. They only learned about periodontal disease and periodontitis after being examined by the clinician at the UKM dental clinic. The lack of awareness may be due to the scarcity of literacy-appropriate information resources. From recent studies, lack of information resources is one of the main causes of poor oral health-related literacy. ${ }^{18}$
Table 2. Video respondent's characteristics

\begin{tabular}{ll}
\hline Variables & N (\%) \\
\hline Age (mean and SD) & $23.5 \pm 11.805$ \\
Highest Education Level & \\
Primary school & $4(6.7)$ \\
Secondary school & $29(48.3)$ \\
College/ university & $27(45)$ \\
Employment status & \\
Working & $30(50)$ \\
Not working & $3(5)$ \\
Student & $27(45)$ \\
Receive treatment at Faculty & \\
Yes & $22(36.7)$ \\
No & $38(63.3)$ \\
\hline
\end{tabular}

Table 3. Respondent's feedback on videos viewed

\begin{tabular}{llll}
\hline Question asked & $\begin{array}{l}\text { Video 1 } \\
\text { N (\%) }\end{array}$ & $\begin{array}{l}\text { Video 2 } \\
\text { N (\%) }\end{array}$ & $\begin{array}{l}\text { Video 3 } \\
\text { N (\%) }\end{array}$ \\
\hline Video is interesting & $54(90)$ & $55(92)$ & $58(97)$ \\
Video is easily understood & $59(98)$ & $59(98)$ & $57(95)$ \\
Information is useful & $60(100)$ & $60(100)$ & $59(98)$ \\
Satisfied with video & $57(95)$ & $57(95)$ & $57(95)$ \\
\hline
\end{tabular}

Table 4. Specific comments about the videos

\begin{tabular}{|c|c|}
\hline 1. & $\begin{array}{l}\text { Duration } \\
\text { Too long } \\
\text { Short and simple is more preferable }\end{array}$ \\
\hline 2. & $\begin{array}{l}\text { Audio-visual quality } \\
\text { Put more pictures about periodontitis } \\
\text { Use mind map to summarise the video } \\
\text { Use more animation } \\
\text { Modify on voice projection } \\
\text { Use variation of voice intonation } \\
\text { The music background was too loud }\end{array}$ \\
\hline 3. & $\begin{array}{l}\text { Medical jargons/ terminologies } \\
\text { Cannot understand medical terms } \\
\text { Used exact words } \\
\text { Use simple word and easily understandable } \\
\text { Provide subtitle }\end{array}$ \\
\hline 4. & $\begin{array}{l}\text { Layout attractiveness } \\
\text { Too much interview } \\
\text { The video is too formal } \\
\text { Nice video and informative } \\
\text { Use more attractive fonts }\end{array}$ \\
\hline 5. & $\begin{array}{l}\text { Need for Elaboration } \\
\text { Give more examples } \\
\text { Include toothbrushing technique and diet counseling } \\
\text { Be specific on the topic discussed } \\
\text { Needs more actors and actresses }\end{array}$ \\
\hline
\end{tabular}

The three videos produced contained information about what the public and patients with periodontal disease would want to know and need to know about periodontitis and diabetes. Sixty participants evaluated the videos. Their mean age was 29 years old with a standard deviation of 11 years. This represents a 
younger adult population and therefore the findings of the video evaluation can be said to be useful for this particular age group. About half of the respondents had received up to secondary school education while a small proportion had received only primary school education. Many were university students. While this does not predict the oral health literacy level it is safe to suggest that the majority of them may have inadequate oral health literacy. This is because studies suggest that even highly educated people can have low levels of health literacy. ${ }^{19}$ According to the findings, there were equal proportions of respondents who were patients at the Dental Faculty and who were the general public. While we may assume that dental patients may have better oral health literacy levels this may not always be the case; the majority of the public will have inadequate oral health literacy as suggested by studies of health literacy - low health literacy will contribute to a person having inadequate oral health literacy. ${ }^{20}$ Consequently the sample who evaluated the videos may be said to represent a younger generation of the public with various levels of oral health literacy. This is an acceptable assumption as it was not the intention of this study to determine the exact oral health literacy levels of these respondents, nor to measure whether there would be improvements of these levels after viewing the videos.

However, this study did set out to evaluate whether the newly-produced videos were considered appropriate and acceptable for people of all oral health literacy levels. There will always be new knowledge after having gone through an educational activity and therefore evaluating increases of knowledge will not be reflective of the video effectiveness. After viewing the videos, about more than $95 \%$ of the respondents agree that they were interesting, easily understood and contained useful information. This conforms with findings of the FGD which suggests that the public prefers to receive health information from multimedia presentation compared to having to read written materials as it is easier to understand and can create their interest in the subject. According to Kandula and co-workers, the combination of sound, graphic and text can reinforce and complement one another to facilitate learning and maintain audience interest. ${ }^{14}$ The use of PEMAT guidelines was particularly useful in ensuring that contents were easily understood for people of various health literacy abilities. ${ }^{17}$ It was heartening to know that the contents were useful as the information was developed to fill the gap of knowledge identified during the FGD.

About 95\% respondents were satisfied with the videos. The remaining who was not satisfied had provided some additional comments. We categorised the comments as the following: duration, audio-visual quality, medical jargons/ terminologies, layout attractiveness and need for elaboration (Table 4). These comments are useful to be utilized in order to improve the videos. Some of the respondents stated that the videos were too lengthy. With at least five minutes long per video, some of them felt that the videos should be shorter in order to retain their attention throughout the viewing session. Next was the audio-visual quality - some respondents suggested that we use more pictures to describe periodontitis. This is because periodontitis is not a well-known disease and public may need more description by means of pictures in order to appreciate how it looks like. They also think that by adding mind maps and animations, they may further understand the videos. Voice intonation was also suggested to be more varied so that it does not sound monotonous. Third aspect is the medical jargons used. The respondents suggested that the video only incorporate layman terms. If medical terms are to be used, they suggest subtitles to be included. In the attractiveness aspect, some of the respondents said that the videos are too formal, with much interviews involved. Some suggested that attractive fonts to be used. However, there are some that even like the videos. In terms of elaboration, some respondents suggested that more examples are to be given in order to make the videos more literacy appropriate. Some even suggested that the videos have to be more specific on the topics discussed.

This study is limited by the convenient sampling of participants for the package evaluation survey which may result in study conclusions that do not represent the whole population. Respondents were generally higher educated and lived in the city, which means that they are more likely to give more positive responses to the survey as compared to those who are socially disadvantaged.

\section{CONCLUSION}

The health education package developed in this study was potentially literacy-appropriate for the general public as the majority of the patients gave positive response. The public may be given access to these videos via online education portals and at both medical and dental clinics waiting areas.

\section{ACKNOWLEDGMENT}

This study was funded by the UKM Strategic/Action Plan Research Grant (PTS 2014-054). The authors are indebted to the patients who were involved with the FGD and filming of the videos. 


\section{REFERENCES}

1. Valerio MA, Kanjirath PP, Klausner CP, Peters MC. A qualitative examination of patient awareness and understanding of type 2 diabetes and oral health care needs. Diabetes Res Clin Pract. 2011;93:15965.

2. Lindenmeyer A, Bowyer V, Roscoe J, Dale J, Sutcliffe P. Oral health awareness and care preferences in patients with diabetes: a qualitative study. Fam Pract. 2013;30:113-8.

3. Yen HK, Wolf BJ, Bandyopadhyay D, Magruder KM, Salinas CF, London SD. Oral health knowledge and behavior among adults with diabetes. Diabetes Res Clin Pract. 2009;86:239-46.

4. National Institute of Dental and Craniofacial Research, National Institute of Health, U.S. Public Health Service, Department of Health and Human Services. The invisible barrier: literacy and its relationship with oral health. A report of a workgroup sponsored by the National Institute of Dental and Craniofacial Research, National Institute of Health, U.S. Public Health Service, Department of Health and Human Services. J Public Health Dent. 2005;65:174-82.

5. Lee JY, Divaris K, Baker AD, Rozier RG, Vann WF Jr. The relationship of oral health literacy and self-efficacy with oral health status and dental neglect. Am J Public Health. 2012;102:923-9.

6. Borgnakke WS, Ylöstalo PV, Taylor GW, Genco RJ. Effect of periodontal disease on diabetes: systematic review of epidemiologic observational evidence. J Periodontol. 2013;84:S135-52.

7. Egebretson S, Kocher T. Evidence that periodontal treatment improves diabetes outcomes: a systematic review and meta-analysis. J Periodontol. 2013;84:S153-69.

8. Löe H. Periodontal disease. The sixth complication of diabetes mellitus. Diabetes Care. 1993;16:32934.

9. Badiah B, Kang PL, Hor WS, Razali M, Dom TNM. Exploring factors influencing adherence to oral hygiene care among periodontal patients: A pilot study. Sains Malaysiana. 2013;42: 13-7.

10. Peshaw PM. Periodontal disease and diabetes. J Dent. 2009;37:S575-7.
11. Dom TNM, Muttalib KA, Ayob R, Yaw SL, Asadi ASM, et al. Periodontal status and provision of periodontal services in Malaysia: trends and way forward. MJPHM. 2013;13:38-47.

12. Ministry of Health, Malaysia. National Health And Morbidity Survey 2011 Fact Sheet; 2011. p. 4-5.

13. Horowitz AM, Kleinman DV. Oral health literacy: a pathway to reducing oral health disparities in Maryland. J Public Health Dent. 2012;72:S26-30.

14. Kandula NR, Nsiah-Kumi PA, Makoul G, Sager J, Zei CP, Glass S. The relationship between health literacy and knowledge improvement after a multimedia type 2 diabetes education program. Patient Educ Couns. 2009;75:321-7.

15. Wallace AS, Seligman HK, Davis TC, Schillinger D, Arnold CL, Bryant-Shilliday B. Literacyappropriate educational materials and brief counseling improve diabetes self-management. Patient Educ Couns. 2009;75:328-33.

16. Stewart K, Gill P, Chadwick B, Treasure E. Qualitative research in dentistry. Br Dent J. 2008;204:235-9.

17. Agency for Healthcare Research and Quality, U.S. Public Health Service. The patient education materials assessment tool (PEMAT) and user's guide. An instrument to assess the understandability and actionability of print and audiovisual patient education materials.- [cited 2015 June 24]. Available from: http://www. ahrq.gov/professionals/prevention-chronic-care/ improve/self-mgmt/pemat/

18. Naghibi Sistani MM, Montazeri A, Yazdani R, Murtomaa H. New oral health literacy instrument for public health: development and pilot testing. J Investig Clin Dent. 2014;5:313-21.

19. Institute of Medicine (US) Committee on Health Literacy; Nielsen-Bohlman L, Panzer AM, Kindig DA, editors. Health Literacy: A Prescription to End Confusion. Washington (DC): National Academies Press (US);2004.

20. National Institute of Dental and Craniofacial Research, National Institute of Health, U.S. Public Health Service, Department of Health and Human Services. The invisible barrier: literacy and its relationship with oral health. J Public Health Dent. 2005;65:174-82.

(Received July 1, 2015; Accepted August 28, 2015) 